This item was submitted to Loughborough's Research Repository by the author.

Items in Figshare are protected by copyright, with all rights reserved, unless otherwise indicated.

\title{
A multidimensional positive definite remapping for Lagrangian solutions of the Noh problem
}

PLEASE CITE THE PUBLISHED VERSION

http://dx.doi.org/10.1016/j.compfluid.2010.09.007

PUBLISHER

(C) Elsevier

VERSION

AM (Accepted Manuscript)

LICENCE

CC BY-NC-ND 4.0

\section{REPOSITORY RECORD}

Hill, Ryan N., and Joanna Szmelter. 2019. "A Multidimensional Positive Definite Remapping for Lagrangian Solutions of the Noh Problem". figshare. https://hdl.handle.net/2134/13367. 
This item was submitted to Loughborough's Institutional Repository (https://dspace.lboro.ac.uk/) by the author and is made available under the following Creative Commons Licence conditions.

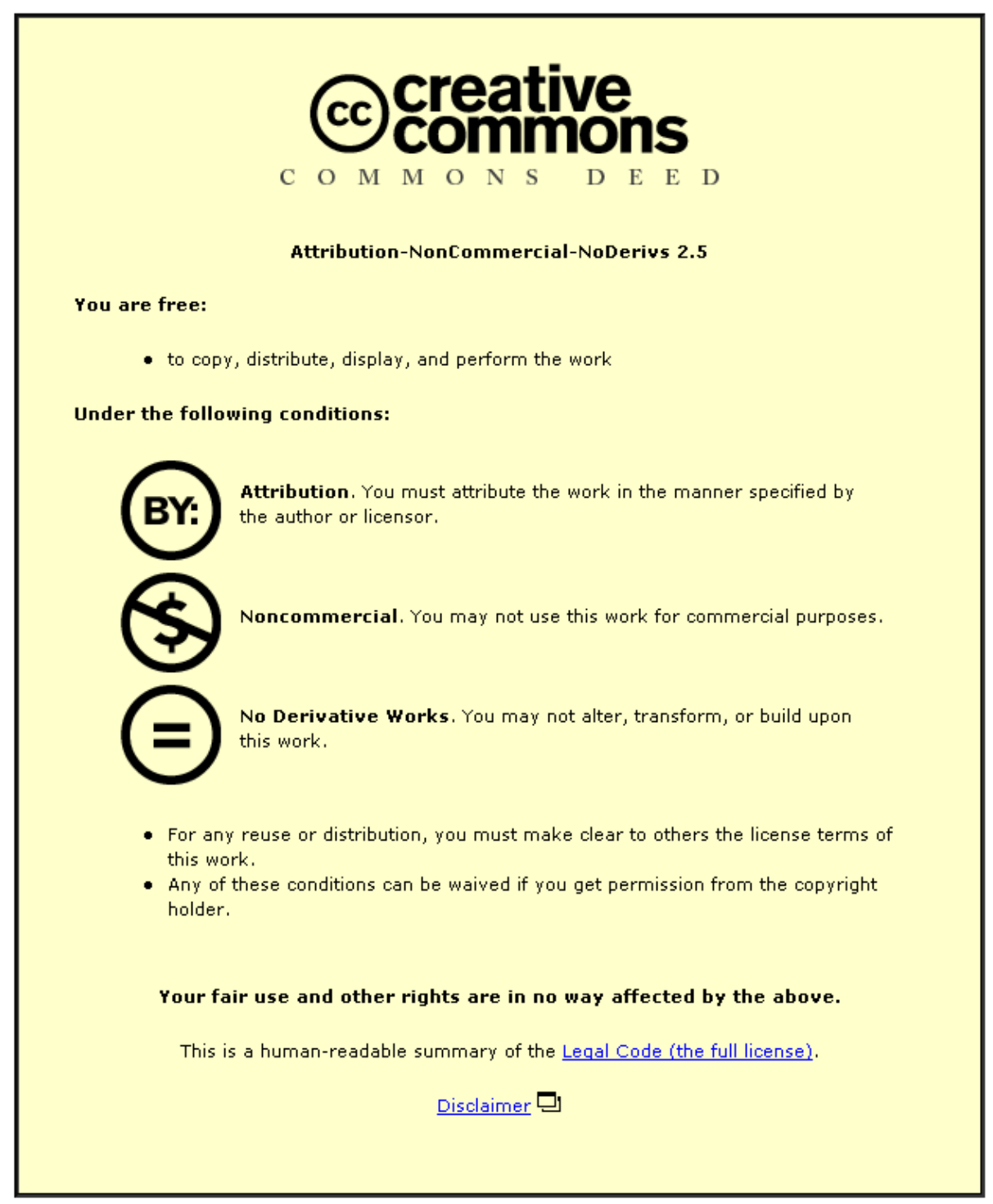

For the full text of this licence, please go to: http://creativecommons.org/licenses/by-nc-nd/2.5/ 


\title{
A Multidimensional Positive Definite Remapping for Lagrangian Solutions of The Noh Problem
}

\author{
Ryan N. Hilla, Joanna Szmelter ${ }^{\mathrm{a}}$ \\ ${ }^{a}$ Loughborough University, Leicestershire, LE11 3TU, UK
}

\begin{abstract}
A remapping based on the multidimensional positive definite advection transport algorithm (MPDATA), implemented for ALE methods, is used to model the Noh problem. Typical solutions in the Lagrangian reference frame contain heating errors which arise during the simulation of a shock reflection originating at a wall. The paper shows that the inherent properties of MPDATA can be exploited in the remapping to reduce wall heating errors. The resulting increase in accuracy and symmetry of solutions is demonstrated. Keywords: ALE remapping, MPDATA gauge, Conservative interpolation, Noh problem
\end{abstract}

\section{INTRODUCTION}

Remapping within an arbitrary Lagrangian-Eulerian (ALE) scheme requires values of a scalar to be conservatively interpolated from one computational mesh to another which has differing geometry. Advection methods are typically utilised for the remapping phase, with fluxes being created by overlapping volumes between adjacent elements. This paper documents the

Email address: j.szmelter@lboro.ac.uk (Joanna Szmelter) 
application of MPDATA $[11,12]$ as a remapping scheme for this purpose.

Demonstrations focus on the classical Noh problem. The problem is relevant to shock reflection and interactions, and has been extensively studied; refer to $[7,8]$ as well as the literature therein for in-depth analysis and reviews. In general, accurate solutions of the Noh problem can be obtained by methods based on the Eulerian framework, but simulations involving Lagrangian solutions introduce unphysical wall heating. ALE methods inherit errors introduced in the Lagrangian phase, which in turn raises an issue for the remapping phase. The remapping phase should accurately interpolate the Lagrangian solution, including all significant features of the variables, whether such features are deemed accurate or otherwise. In the case of the Noh problem, the wall heating features are unphysical, and should not be present in the solution. The remapping phase may then be employed to repair the solution in a computationally efficient manner.

This study describes an MPDATA based treatment for reducing the wall heating errors in ALE/Lagrangian-Eulerian calculations, and highlights additional benefits of this treatment, such as the restoration of symmetry in axisymmetric test cases.

\section{MPDATA Remapping}

The use of MPDATA inspired methods for the conservative interpolation of scalar fields with prescribed mesh movement has been documented in [6] and has recently been followed by the development of MPDATA based remapping for ALE methods [5]. The implementation in [5] and in this

work uses the ALE scheme described in $[1,2]$. The ALE scheme consists 


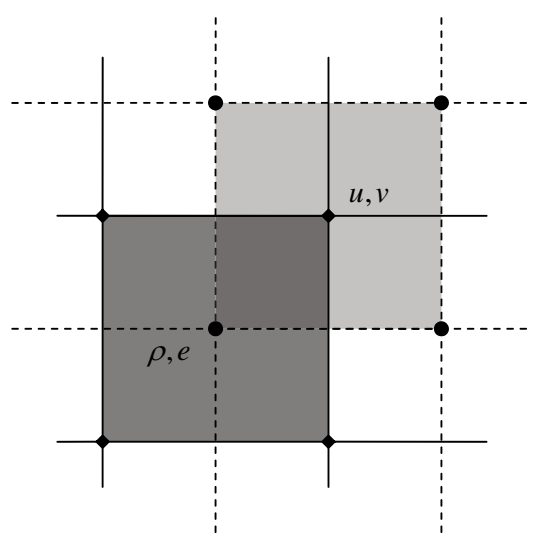

Figure 1: Illustration of the staggered grid arrangement. Density, $\rho$, and internal energy, $e$, are stored at element centres (circles) on the computational mesh (solid lines). Components of velocity, $u$ and $v$, are stored at nodes (diamonds) which become the centres of elements on the dual mesh (dashed lines).

of a Lagrangian phase which moves the material and computational mesh, a rezone phase to define a mesh with improved geometry, and an Eulerian remap phase to transport the material from the Lagrangian mesh to the rezoned mesh. A dual mesh based procedure is required for the momentum remap to account for the staggered nature of the ALE scheme, as described in $[1,3,5]$ whereby element masses and mass fluxes are equally distributed to all nodes of the element. The arrangement of variables is shown in Figure 1. Consequently, density is remapped in volume coordinates [3]; and internal energy (without total energy conservation compensation) and components of velocity are remapped using mass coordinates. The mass coordinate scheme differs technically from the volume coordinate scheme only in the detail that volumes are replaced by element masses in the calculations. The MPDATA remapping routine remains the same for both scalar and vector variables. 


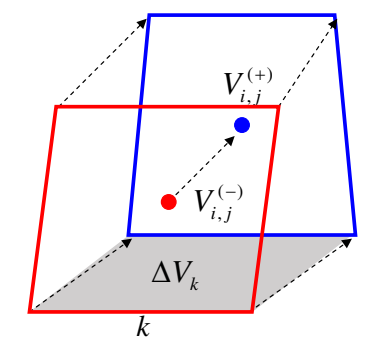

Figure 2: Definition of volumes on old (red) and new (blue) grids, with illustration of overlap volumes, $\Delta V_{k}$.

In all examples presented here, the ALE algorithm operates as an Eulerian scheme whereby the rezone phase restores the initial computational mesh after each Lagrangian calculation. An alternate remapping scheme based upon the second order van Leer advection algorithm is available with the same ALE algorithm, and is used for comparison throughout the paper. For examples using the full ALE algorithm with MPDATA remapping, see [5].

\section{MPDATA Based Remapping with Second-Order Filtering}

In order to use MPDATA for remapping, it is useful to utilise the volume coordinate update of a scalar. For arbitrary flows, the volume update, as illustrated in Figure 2, is given as

$$
V^{(+)}=V^{(-)}-\Sigma_{k=1}^{4} \Delta V_{k},
$$

where $\Delta V_{k}$ denotes the quadrilateral generated by the movement of edge $k$ from the post-Lagrangian mesh, denoted ${ }^{(-)}$, to the remapped mesh, denoted ${ }^{(+)}$. The scalar in each cell is given by its mean value over the cell volume, so that the corresponding scalar update is then

$$
\psi^{(+)}=\frac{V^{(-)} \psi^{(-)}}{V^{(+)}}-\frac{1}{V^{(+)}} \Sigma_{k=1}^{4} \Delta V_{k} \psi_{k}^{(-)} .
$$


Equation (2) corresponds to the first order upwind calculation required for MPDATA remapping. Upwind advection utilises a Courant number that is also required for the calculation of the pseudo velocities used to compensate the second order truncation error in MPDATA. Identification of the Courant number within (2) is therefore necessary for the subsequent steps in the algorithm.

Defining $\Psi:=V \psi$ and multiplying (2) by $V^{(-)} / V^{(-)}$, the scalar remapping becomes

$$
\Psi^{(+)}=\Psi^{(-)}-\Sigma_{k=1}^{4} \frac{\Delta V_{k}}{V^{(-)}} \Psi_{k}^{(-)},
$$

which is now in the form of upwind advection used in [10], with $\Delta V_{k} / V^{(-)}$ being akin to the Courant number evaluated at edge $k$ of an element ${ }^{1}$. For consistency, the volume in the denominator of (3) is obtained by averaging over element volumes on either side of the edge. Repeating the update (2)-(3) using mass instead of volumes reveals that $\Delta V / V^{(-)}$can be interpreted as the Courant number for all scalar and vector variables. The MPDATA scheme, generalised to volume coordinates including second-order filtering [13] is summarised below. Following [10], which provides the detailed derivation of MPDATA, only the volume coordinate extension is provided here, and to assist the reader, notation adopted from [10] is used.

By denoting the Courant number by $C=\Delta V / V^{(-)}$, the first pass is

\footnotetext{
${ }^{1}$ This interpretation of the Courant number follows a more formal derivation of the upwind scheme from the scalar update in volume coordinates, and differs from the interpretation given in [5].
} 
formulated as

$$
\begin{aligned}
\Psi_{i, j}^{(1)}=\Psi_{i, j}^{(-)} & -\left[F\left(\Psi_{i, j}^{(-)}, \Psi_{i+1, j}^{(-)}, C_{i+1 / 2, j}\right)-\beta_{i+1 / 2, j}\left(\Psi_{i+1, j}^{(-)}-\Psi_{i, j}^{(-)}\right)\right. \\
& \left.-F\left(\Psi_{i-1, j}^{(-)}, \Psi_{i, j}^{(-)}, C_{i-1 / 2, j}\right)-\beta_{i-1 / 2, j}\left(\Psi_{i, j}^{(-)}-\Psi_{i-1, j}^{(-)}\right)\right] \\
& -\left[F\left(\Psi_{i, j}^{(-)}, \Psi_{i, j+1}^{(-)}, C_{i, j+1 / 2}\right)-\beta_{i, j+1 / 2}\left(\Psi_{i, j+1}^{(-)}-\Psi_{i, j}^{(-)}\right)\right. \\
- & \left.F\left(\Psi_{i, j-1}^{(-)}, \Psi_{i, j}^{(-)}, C_{i, j-1 / 2}\right)-\beta_{i, j-1 / 2}\left(\Psi_{i, j}^{(-)}-\Psi_{i, j-1}^{(-)}\right)\right],
\end{aligned}
$$

where half integer index subscripts indicate edge (and nodal) centred variables, and integer indices denote element centred variables. The flux function $F$ is defined as

$$
F\left(\Psi_{a}, \Psi_{b}, C\right) \equiv \frac{1}{2}(C+|C|) \Psi_{a}+\frac{1}{2}(C-|C|) \Psi_{b}
$$

Terms utilising the small positive coefficient $\beta$ are activated when oscillations are present in the first-order solution and may be filtered.

The pseudo velocities (antidiffusive overlap volumes), modified into antidiffusive Courant numbers for the second pass are

$$
C_{i+1 / 2, j}^{(1)} \equiv\left(\left|C_{i+1 / 2, j}\right|-\left(C_{i+1 / 2, j}\right)^{2}+2 \beta_{i+1 / 2, j}\right) A^{(1)}-C_{i+1 / 2, j} \bar{C}_{i+1 / 2, j} B^{(1)},
$$

where $\bar{C}_{i+1 / 2, j}=1 / 4\left(C_{i, j+1 / 2}+C_{i, j-1 / 2}+C_{i+1, j+1 / 2}+C_{i+1, j-1 / 2}\right)$, with an analogous expression for $C_{i-1 / 2, j}^{(1)}$, and

$C_{i, j+1 / 2}^{(1)} \equiv\left(\left|C_{i, j+1 / 2}\right|-\left(C_{i, j+1 / 2}\right)^{2}+2 \beta_{i, j+1 / 2}\right) B^{(1)}-\bar{C}_{i, j+1 / 2} C_{i, j+1 / 2} A^{(1)}$,

where $\bar{C}_{i, j+1 / 2}=1 / 4\left(C_{i+1 / 2, j}+C_{i-1 / 2, j}+C_{i+1 / 2, j+1}+C_{i-1 / 2, j+1}\right)$, with an analogous expression for $C_{i, j-1 / 2}^{(1)}$; where

$$
A^{(1)} \equiv\left[\frac{\delta x}{2 \psi} \frac{\partial \psi}{\partial x}\right]_{i+1 / 2, j}^{(+)}=\frac{\left|\psi_{i+1, j}^{(1)}\right|-\left|\psi_{i, j}^{(1)}\right|}{\left|\psi_{i+1, j}^{(1)}\right|+\left|\psi_{i, j}^{(1)}\right|}
$$




$$
B^{(1)} \equiv\left[\frac{\delta y}{2 \psi} \frac{\partial \psi}{\partial y}\right]_{i+1 / 2, j}^{(+)}=\frac{1}{2} \frac{\left|\psi_{i+1, j+1}^{(1)}\right|+\left|\psi_{i, j+1}^{(1)}\right|-\left|\psi_{i+1, j-1}^{(1)}\right|-\left|\psi_{i, j-1}^{(1)}\right|}{\left|\psi_{i+1, j+1}^{(1)}\right|+\left|\psi_{i, j+1}^{(1)}\right|+\left|\psi_{i+1, j-1}^{(1)}\right|+\left|\psi_{i, j-1}^{(1)}\right|} .
$$

The corrective pass then takes the following form,

$$
\begin{aligned}
\Psi_{i, j}^{(+)}=\Psi_{i, j}^{(1)} & -\left[F\left(\Psi_{i, j}^{(1)}, \Psi_{i+1, j}^{(1)}, C_{i+1 / 2, j}^{(1)}\right)-F\left(\Psi_{i-1, j}^{(1)}, \Psi_{i, j}^{(1)}, C_{i-1 / 2, j}^{(1)}\right)\right] \\
- & {\left[F\left(\Psi_{i, j}^{(1)}, \Psi_{i, j+1}^{(1)}, C_{i, j+1 / 2}^{(1)}\right)-F\left(\Psi_{i, j-1}^{(1)}, \Psi_{i, j}^{(1)}, C_{i, j-1 / 2}^{(1)}\right)\right], }
\end{aligned}
$$

The infinite gauge (whereby the algorithm is linearised around an arbitrarily large constant) and monotonic options used within the remapping in this paper are detailed in [10] and [12] respectively. In Appendix A, a study of the MPDATA remapping shows that in the context of ALE schemes, the infinite gauge option is more general and accurate than the basic MPDATA.

\section{The Noh Problem}

The Noh problem [7] consists of a cold, ideal gas with density $\rho=1.0$, internal energy $e=0.0$, ratio of specific heat $\gamma=\frac{5}{3}$ and uniform velocity $\|\mathbf{v}\|=-1.0$ forcing the gas into a rigid wall. An infinite strength shock is generated at the wall boundary and travels in the opposite direction to the gas flow. The simulation of the planar case (rigid wall along $x=0.0$ ) is performed in a $[0,1] \times[0,0.2]$ domain, discretised with a series of uniform resolution grids. The simulation of the axisymmetric case (wall at $(x, y)=(0,0))$ is performed on a Cartesian mesh on a $[0,1] \times[0,1]$ domain, with $200 \times 200$ elements, and $\Delta t=0.0005$. Exact values are used on inflow boundaries, with symmetry utilised on boundaries $x=0$ and $y=0$ in the axisymmetric case. Both planar and axisymmetric cases use a constant time step, terminating at time $t=0.6$. 
The wall heating error in the Noh problem arises in the Lagrangian solution of unsteady wave propagation and is related to factors such as the application of artificial viscosity, phase errors, wave speed or changes in mesh resolution travelling with the shock. A thorough analysis of the sources of wall heating is provided in Reference [8]. Due to the nature of the Noh problem, a strong shock is formed next to an impermeable wall. At this stage, elements near the boundary experience large compression so that the added artificial viscosity cannot be dissipated sufficiently, manifesting itself as unphysical heating. This generates a build up of energy at the wall boundary which in turn forces a drop in the density as the equation of state establishes the correct pressure level. Herein, properties of the MPDATA based remapping are exploited to regulate the solution.

By design, MPDATA relies on the iterative application of the upwind scheme, where subsequent iterations compensate for the implicit viscosity of the preceding steps. Thus it bears an analogy to generalised similarity models, where an estimate of the full unfiltered Navier-Stokes velocity (that enters the subgrid-scale stress tensor) is obtained by an approximate inversion of the filtering operation, i.e. deconvolution [11]. Building upon this concept, and in the spirit of Flux Corrected Transport methods [14], additional diffusion i.e. activating $\beta$ terms in (4)-(6) may be explicitly added to the first upwind iteration to remove oscillations in the first-order solution. The second iteration, (9), compensates the truncation error of the first step (4) which includes the added dissipation. With $\delta t \propto \delta x$, the explicit diffusion enters (4) as an $\mathcal{O}(\delta x)$ correction, whereupon its reversal in the corrective step leaves an $\mathcal{O}\left(\delta x^{2}\right)$ residual; see [13] for a thorough exposition. Similarly 

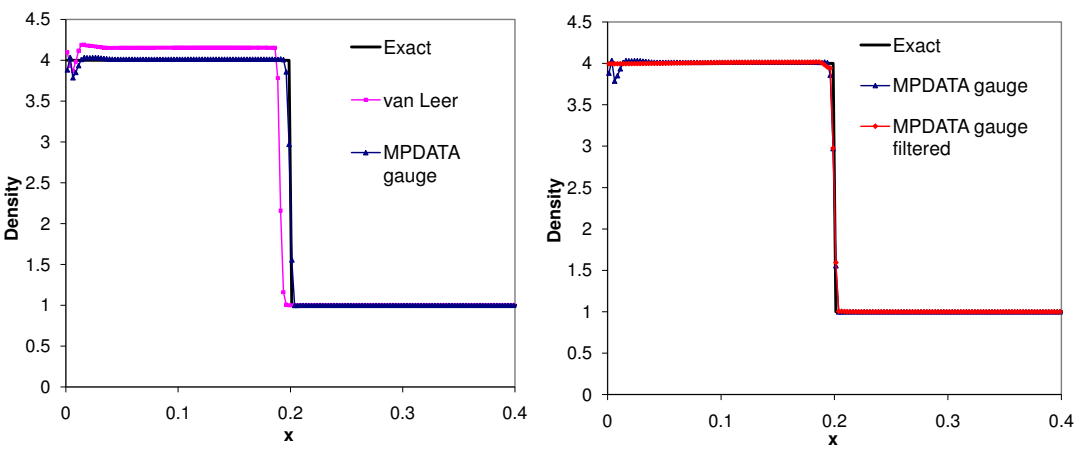

Figure 3: Density profiles of the planar case of the Noh problem $(400 \times 20, \Delta t=0.00025)$.

to MPDATA, this treatment is fully multidimensional.

Within the second-order filtering option, $\beta=0.02$ is the default value, but may vary in space or be set to zero upon the detection of a shock (discontinuity). The effective level of diffusion is an order of magnitude lower than that reported in [14]. Wall heating errors will be the focus of this study, with preservation of symmetry receiving attention in the axisymmetric case.

\subsection{Planar Case}

The left plate of Fig. 3 shows the density profile for the van Leer and MPDATA-gauge $(\beta=0.0)$ schemes. It can be seen in this diagram that the MPDATA result is correctly aligned to the exact solution in terms of the shock position ( $x=0.19958$ using linear interpolation for $\rho=2.5)$ and the level of post-shock density accumulation, whereas the van Leer scheme incorrectly aligns both features (shock at $x=0.19073$ ) in a manner consistent with results shown in [8] for Eulerian calculations using internal energy.

The van Leer scheme has masked the wall heating error. This feature has arisen due to the van Leer scheme being forced to give a first-order accurate solution at the wall elements because the larger stencil required to construct 


\begin{tabular}{ccccccc} 
& Grid & Density & I.E. & T.E. & Pressure & Velocity \\
\hline van Leer & $100 \times 20$ & 0.105941 & 0.148222 & 0.148211 & 0.186724 & 0.079659 \\
& $200 \times 20$ & 0.126266 & 0.188564 & 0.188557 & 0.203178 & 0.094454 \\
& $400 \times 20$ & 0.139633 & 0.208208 & 0.208204 & 0.212429 & 0.102806 \\
\hline MPDATA & $100 \times 20$ & 0.058166 & 0.064287 & 0.064252 & 0.074276 & 0.035359 \\
& $200 \times 20$ & 0.040818 & 0.043834 & 0.043832 & 0.058089 & 0.025558 \\
& $400 \times 20$ & 0.030565 & 0.035261 & 0.035260 & 0.053451 & 0.021844 \\
\hline MPDATA & $100 \times 20$ & 0.056939 & 0.061482 & 0.061475 & 0.075888 & 0.035611 \\
filtered & $200 \times 20$ & 0.039963 & 0.042083 & 0.042081 & 0.057768 & 0.025556 \\
& $400 \times 20$ & 0.029813 & 0.033872 & 0.033871 & 0.053052 & 0.021781 \\
\hline
\end{tabular}

Table 1: $L_{2}$ error data for planar Noh problem with increasing mesh resolution in the direction of flow. I.E. denotes internal energy; T.E., total energy.

the monotonic piecewise linear distribution of the transported variables is not available at the boundary. The MPDATA based scheme has accurately remapped the Lagrangian wall heating features, which are unphysical, near the boundary at $x=0.0$, therefore a self-regulating application of small explicit dissipation of wall heating errors is beneficial. This is achieved with the second-order filtering option $(\beta=0.02$ for density and internal energy remapping) which removes the build up of energy at the boundary.

The filtering option of MPDATA is applied anisotropically in the direction of flow at all element edges except those where a shock has been detected. Therefore, the filtering does not smear the shock any further than has been done so with the application of artificial viscosity in the Lagrangian phase, and gives a more accurate shock position $(x=0.19961)$. The shock is de- 


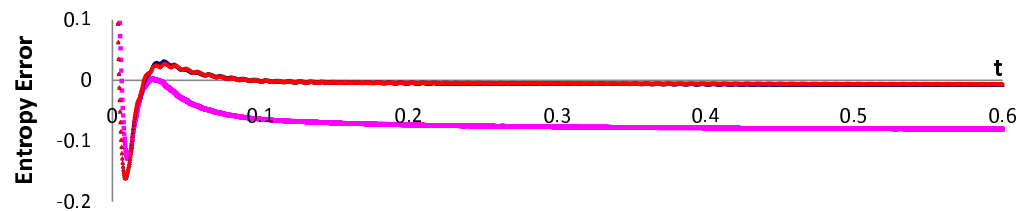

Figure 4: Entropy errors for the planar Noh Problem $(400 \times 20, \Delta t=0.00025)$. van Leer (pink square), MPDATA gauge (blue triangle), MPDATA gauge filtered (red diamond).

tected automatically by examination of pressure gradients. The exclusion of the shock from the filtering is not essential, but provides a modest enhancement.

The behaviour, illustrated in Figure 3, of all schemes is consistent across coarse and finer resolution meshes. The departures from the exact solutions are reflected by Table 1 which gives $L_{2}$ error data for $100 \times 20,200 \times 20$ and $400 \times 20$ meshes. These norm values show consistently higher errors in all variables for the van Leer scheme compared to the MPDATA based scheme. Due to the presence of a shock, the treatment of inflow boundaries and other factors, Table 1 is not suitable for an assessment of asymptotic mesh convergence. A second order asymptotic mesh convergence study of MPDATA remapping was conducted in [5] for a pure advection test with prescribed mesh velocity.

Solutions using the internal energy equation depend upon the level of entropy production. Fig. 4 shows the ratio of entropy error (defined in [8]) for each method. All methods have large start up errors (maximum entropy error for the van Leer scheme is 1.652, MPDATA based schemes, 1.655), however the van Leer scheme stabilises to a level significantly below the correct production level so that the incorrect features are evident. The MPDATA 

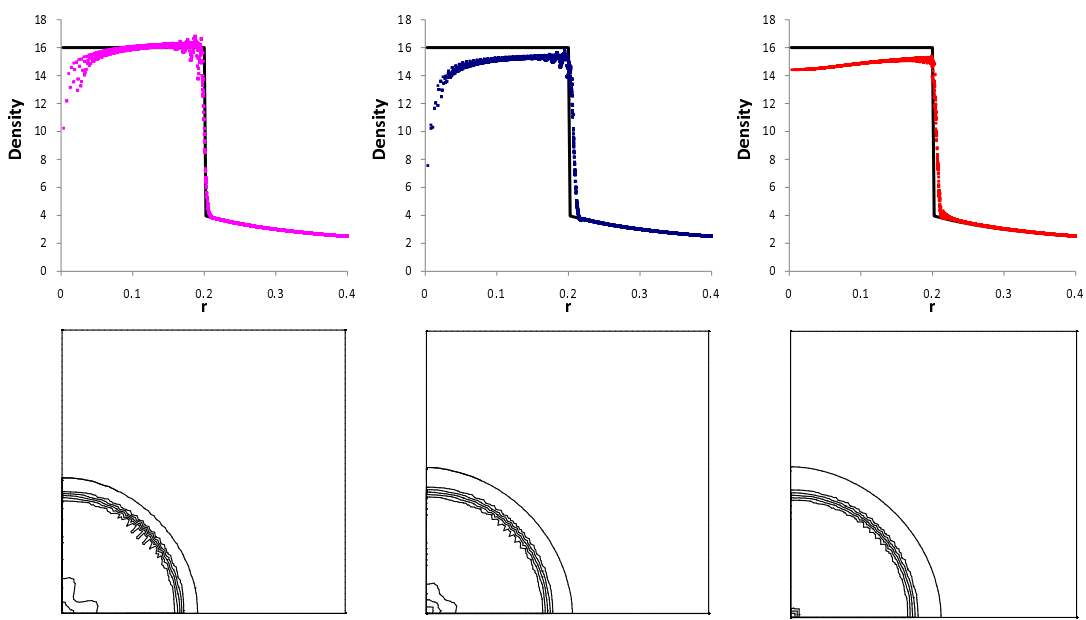

Figure 5: Upper row: Density plotted against radius for the axisymmetric case of the Noh problem (Cartesian mesh). Left: van Leer, centre: MPDATA gauge, right: MPDATA gauge with second order filtering. Lower row: Corresponding density contours (quarter of the domain shown), minimum value $\rho=2.0$, contour interval, 1.0.

solutions attain more accurate levels of entropy production, however it can be seen that the start up errors and the conservation of internal energy rather than total energy, cf. [8, 13], are still affecting the solutions. This is seen by the MPDATA-based remapping entropy errors asymptotically approaching a negative value $(-0.006$ at $t=0.6)$.

\subsection{Axisymmetric Case}

Figure 5 shows the density distributions obtained on a $200 \times 200$ Cartesian mesh at $t=0.6(\Delta t=0.0005)$. The van Leer based scheme is extended to two-dimensions by isotropic remapping. In this case corner coupling errors dominate the van Leer solution with a loss of symmetry, and significant errors along the cut $x=y$ (linearly interpolated shock position along for $\rho=10$ 
at $r=0.19932)^{2}$. As in the planar case, the method features a first-order solution at the "wall" (origin), however in this case the first-order solution does not mask the wall heating errors. The MPDATA gauge solution features an incorrect shock position $(r=0.20646)$ because of the wall heating errors. This in turn leads to an under evaluation of post-shock density accumulation. The multidimensional nature of MPDATA provides greater preservation of symmetry, with a significant reduction in errors along the cut $x=y$, as highlighted in the contour plots of Fig. 5.

Applying the second-order filtering with MPDATA gauge significantly reduces the wall heating errors, and improves the preservation of symmetry. However, the effect of filtering is ultimately limited by the underlying Lagrange and MPDATA gauge solution, so that the shock position $(r=0.20544)$ and post-shock density accumulation are improved, but not fully regulated to the correct levels. The key result from the second-order filtering solution in the axisymmetric case is the marked improvement in symmetry.

Christensen [4] monotonic artificial viscosity is used in Lagrangian phase of the simulations, with coefficients $c_{q}=0.75$ and $c_{l}=0.5$. The $c_{l}$ coefficient determines the diffusivity of the artificial viscosity. A reduction of this coefficient results in oscillations along the post shock density accumulation, and is therefore not appropriate for unfiltered methods. The filtering option

\footnotetext{
${ }^{2} \mathrm{~A}$ multidimensional isotropic extension of any one-dimensional scheme is not optimal. A scheme in which a one-dimensional version of MPDATA was employed isotropically in place of the van Leer scheme produced results (not shown) very close to those in the left panel of Fig. 5
} 
however, introduces a small amount of diffusion in the remapping phase, and in this case $c_{l}$ may be reduced, with $c_{q}$ remaining unchanged (not shown). In the planar case, this permits very low wall heating errors on coarse meshes. In the axisymmetric case, greater symmetry preservation is provided due to a reduction of oscillations in the post-shock region near the jump in density, particulary along the cut $x=y$.

\section{Conclusions}

A second-order accurate MPDATA remapping has been presented. The work builds upon developments of the algorithm for ALE calculations shown in [5]. A more rigorous analysis of the relationship between scalar remapping in volume coordinates and upwind advection identified an improved interpretation of the overlap volume based dimensionless quantity which is akin to the Courant number, leading to enhanced accuracy in the remapped solutions. Conservativity and beneficial properties of the infinite gauge option for MPDATA based remapping have been discussed in Appendix A. The infinite gauge option has been shown to correctly deal with distributions containing a change in sign. This property affirms the conclusions obtained in [5] stating that the infinite gauge option offers greater accuracy and flexibility compared to the basic MPDATA for remapping. Improvements in the accuracy of remapped solutions is also obtained by exploiting the properties of MPDATA to preserve symmetry and to remove wall heating errors

MPDATA has shown the ability to retain second order accurate calculations for elements along the wall (or potentially, a material) boundary. The capability of the MPDATA based remapping scheme to correctly remap the 
Lagrangian features at the wall boundary (with or without second-order filtering) is particularly desirable for the application of MPDATA based methods in future multimaterial ALE simulations, in which case the material boundary will have properties similar to a wall boundary.

Acknowledgments: Discussions with Andrew J. Barlow and Robert Kevis are gratefully acknowledged. Comments from anonymous reviewers helped to improve the presentation. This work was supported by the Atomic Weapons Establishment (AWE), Aldermaston.

\section{Appendix A. Sign Changing Remapping}

MPDATA was originally derived for non-negative scalar and vector fields, and subsequently extended to positive or negative fields, cf. Section 3.2 in [10]. This extension utilises absolute values in the calculations of derivatives in the pseudo velocity calculation. However, for some test cases involving fields of varying sign, aspects of the solution may not be accurate. For example, if $\psi_{i, j} \approx-\psi_{i+1, j}$ then $\partial \psi / \partial \mathbf{x} \rightarrow \pm \infty$ as $\psi_{i, j}$ increases in magnitude with $-\psi_{i+1, j}$, whereas $\partial|\psi| / \partial \mathbf{x} \rightarrow 0$. The infinite gauge option of MPDATA is an alternative approach to generalising MPDATA to fields of varying sign.

The volume coordinate infinite gauge option is obtained by linearising the algorithm (4)-(9) around an arbitrarily large constant, see Section 4 in [9] for a detailed discussion of the process. In this way, the scalar or vector field is modified to be effectively non-negative, which in turn removes the need to exploit absolute values in derivatives. In practice, the basic algorithm is altered such that unity replaces each $\Psi$ in the denominators of (7) and (8), and in the fluxes of the corrective pass (9). 

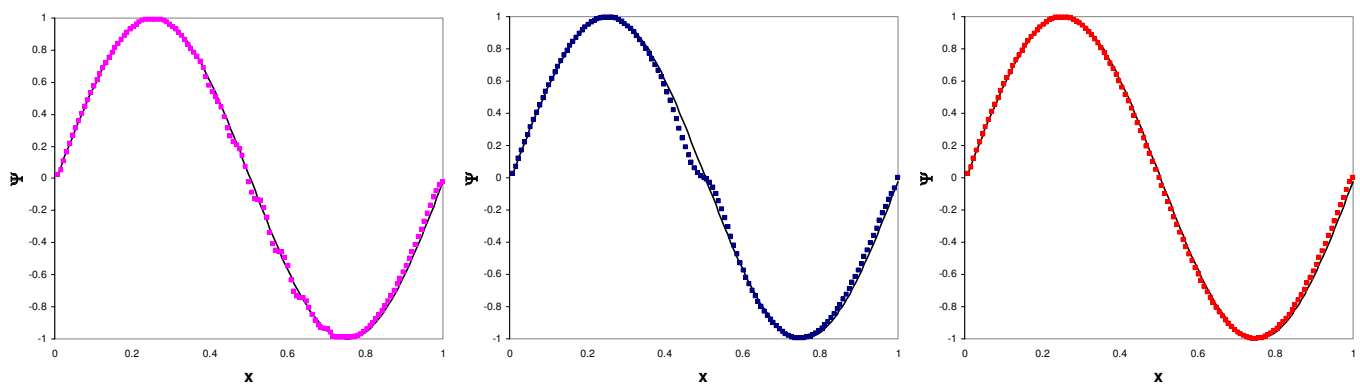

Figure A.6: Profiles of the 'sine' distribution through $x=0.25$ after 640 pseudo timesteps. Left: Isotropic van Leer, center: MPDATA, Infinite gauge MPDATA.

To highlight the benefits of the infinite gauge option, a simple test is considered using the cyclic remapping mesh movement of [6], with a scalar distribution given by

$$
\psi(x, y)=\sin (2 \pi x) \sin (2 \pi y)
$$

remapped onto a series of meshes which are determined by a tensor product mesh movement, as described in [6]. The prescribed mesh movement has the effect of skewing an initially Cartesian mesh, before returning to the initial mesh. The scalar (A.1) reaches a maximum value $\psi=1$, and minimum value $\psi=-1$, and it's distribution features a smooth and continuous change in sign. The test is performed on a $[0,1] \times[0,1]$ domain with $128 \times 128$ elements, using 640 pseudo time steps. Filtering is not employed, i.e. $\beta=0.0$ for both the basic MPDATA and infinite gauge remapping.

Figure A.6 shows a cut through the distribution along $x=0.25$. The isotropic van Leer scheme, shown for comparison, struggles with the mesh movement, and as a result introduces undesired ripples similar to those reported in [5] which dominate the solution (particularly along the profile $x=0.5$, not shown). In contrast, the basic MPDATA solution cor- 


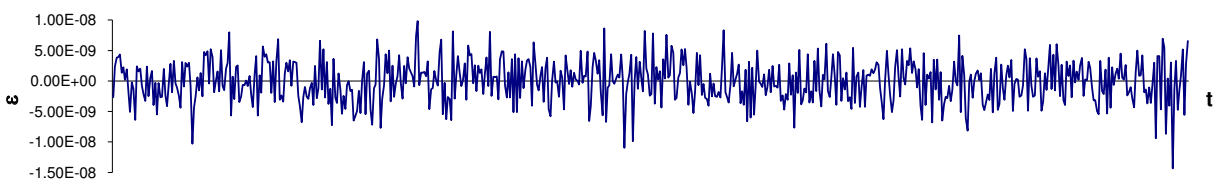

Figure A.7: Temporal evolution of the conservation error for MPDATA gauge remapping.

rectly remaps the scalar, with the exception of regions where the distribution changes sign because $\partial|\psi| / \partial \mathbf{x} \rightarrow 0$. In this case, an unphysical reduction in the gradient of the scalar distribution is introduced. Such errors are not present in the infinite gauge MPDATA solution, with correct remapping achieved for all values. Scalar conservation during the MPDATA gauge calculation, using the error norm

$$
\epsilon=\frac{\sum_{i, j} \Psi_{i, j}^{(+)}-\sum_{i, j} \Psi_{i, j}^{(-)}}{\sum_{i, j} \Psi_{i, j}^{(-)}}
$$

after each pseudo time step, is shown in Figure A.7.

[1] Barlow AJ. An Adaptive Multi-Material Arbitrary Lagrangian Eulerian Algorithm for Computational Shock Hydrodynamics. Ph.D. Thesis, University of Wales, 2002: Swansea.

[2] Barlow AJ. A compatible finite element multi-material ALE hydrodynamics algorithm. Int J Numer Methods Fluids 2008;56:953-64.

[3] Benson DJ. Computational methods in Lagrangian and Eulerian hydrocodes. Comput Meth Appl Mech Eng 1992;99:235-394.

[4] R.B. Christensen. Godunov Methods on a Staggered Mesh - An Improved Artificial Viscosity. Technical Report UCRL-JC-105269 Preprint, Lawrence Livermore National Laboratory, 1990. 
[5] Hill RN, Szmelter J. A Multidimensional Positive Definite Remapping Algorithm for Arbitrary Lagrangian-Eulerian Methods. Int J Numer Methods Fluids 2010, In press, DOI: 10.1002/fld.2351.

[6] Margolin LG, Shashkov M. Second-order sign-preserving conservative interpolation (remapping) on general grids. J Comp Phys 2003;184:26698.

[7] Noh WF. Errors for calculations of strong shocks using an artificial viscosity and an artificial heat flux. J Comp Phys 1987;72:78-120.

[8] W.J. Rider. Revisiting Wall heating, J Comp Phys 2000;162:395-410.

[9] Smolarkiewicz PK, Clark TL. The Multidimensional Positive Definite Advection Transport Algorithm: Further Development and Applications. J Comp Phys 1986;67:396-438.

[10] Smolarkiewicz PK, Margolin LG. MPDATA: A finite-difference solver for geophysical flows. J Comp Phys 1998;140:459-80.

[11] Smolarkiewicz PK. Multidimensional Positive Definite Advection Transport Algorithm: An Overview. Int J Numer Methods Fluids 2006;50:1123-44.

[12] Smolarkiewicz PK, Szmelter J. MPDATA: An edge-based unstructuredgrid formulation. J Comp Phys 2005;206:624-49.

[13] Smolarkiewicz PK, Szmelter J. Iterated Upwind Schemes for Gas Dynamics. J Comp Phys 2009;228:33-54. 
[14] S.T. Zalesak. A fully multidimensional flux-corrected transport algorithm for fluids, J Comp Phys 1979;31:335-62. 